\title{
Search for new physics in lepton + MET final states
}

\author{
Philipp MILLET* \\ (RWTH-Aachen University (DE)) \\ E-mail: philipp.nicolas.milletecern.ch
}

on behalf of the CMS collaboration

\begin{abstract}
A search for new physics in final states with an electron or a muon and a neutrino is presented. This analysis uses 2012 data corresponding to an integrated luminosity of $20 \mathrm{fb}^{-1}$, collected using the CMS detector in pp collisions at a center-of-mass energy of $8 \mathrm{TeV}$ at the LHC. No significant excess of events above the standard model expectation is found in the transverse mass distribution of the lepton-neutrino system. Mass exclusion limits up to $3.35 \mathrm{TeV}$ at $95 \% \mathrm{CL}$ for a SM-like $\mathrm{W}^{\prime}$ boson are determined. Limits are also set on a model where interference between a potentially left-handed $\mathrm{W}^{\prime}$ and the SM W boson occurs. Another interpretation is performed in terms of compositeness, which would manifest itself as a four-fermion contact interaction, setting a limit on the new contact interaction scale $\Lambda$ of $13.0(10.9) \mathrm{TeV}$ for the electron (muon) channel.
\end{abstract}

XXI International Workshop on Deep-Inelastic Scattering and Related Subjects 22-26 April, 2013

Marseilles, France

${ }^{*}$ Speaker. 
A search for new physics in events with a charged lepton (an electron or muon) and a low mass neutrino in the final state has been performed with the Compact Muon Solenoid (CMS) detector [1] at the LHC. The dataset corresponds to an integrated luminosity of $20 \mathrm{fb}^{-1}$ of proton-proton collision data collected in 2012 at a center-of-mass energy of $8 \mathrm{TeV}$. The interpretation of the results is provided in the context of two theoretical models, the sequential standard model $\mathrm{W}^{\prime}$ and a four-fermion contact interaction. This document is based on the results presented in [2]. More information about the analysis strategy can be found there.

New heavy gauge bosons such as $\mathrm{W}^{\prime}$ and $\mathrm{Z}^{\prime}$ are predicted by various extensions of the standard model (SM). In the sequential standard model (SSM) [3], the $\mathrm{W}^{\prime}$ boson is considered to be a heavy analogue of the $\mathrm{W}$. It is assumed to be a narrow resonance with decay modes and branching fractions similar to those of the $\mathrm{W}$, with the addition of the $t \overline{\mathrm{b}}$ channel that becomes relevant for $\mathrm{W}^{\prime}$ masses above $180 \mathrm{GeV}$. If the $\mathrm{W}^{\prime}$ is heavy enough to decay to top and bottom quarks, the predicted branching fraction is about $8.5 \%$ for each of the two leptonic channels studied in the present analysis. Under these assumptions, the width of a $1 \mathrm{TeV} \mathrm{W}^{\prime}$ is about $33 \mathrm{GeV}$. Decays of the $\mathrm{W}^{\prime}$ into $\mathrm{WZ}$ di-bosons are usually suppressed in this model. The assumptions of the SSM were used in previous searches in leptonic channels at the Tevatron [4, 5] and the LHC $[6,7,8,9,10,11]$. If the $\mathrm{W}^{\prime}$ is right-handed it will not interfere with the $\mathrm{W}$ and its signature will be a Jacobian peak, similar to the SM W but at much higher masses. With increasing $\mathrm{W}^{\prime}$ masses the phase space for such heavy particles in pp collisions at $\sqrt{s}=8 \mathrm{TeV}$ decreases, leading to a growing fraction of particles that are produced off-shell at lower masses. If the $\mathrm{W}^{\prime}$ is left-handed, interference with the $\mathrm{W}$ is expected $[12,13,14]$. Constructive (destructive) interference occurs in the mass range between $\mathrm{W}$ and $\mathrm{W}^{\prime}$ if the coupling of the $\mathrm{W}^{\prime}$ boson to quarks and leptons has opposite sign to (same sign as) the coupling of the $\mathrm{W}$ boson to left-handed fermions. In this paper, these models are labelled as SSMO and SSMS for opposite and same sign, respectively. Figure 1-left shows the transverse mass distribution for a $\mathrm{W}^{\prime}$ of $2.4 \mathrm{TeV}$ mass for the cases of constructive (SSMO), destructive (SSMS) and no interference, along with the background due to the SM W.

Another interpretation is possible in terms of a four-fermion contact interaction. Motivated by the observation of mass hierarchies in the fermion sector, the basic model assumption is that quarks and leptons are composite objects of fundamental constituents [15]. At energies much lower than their binding energy, typically called $\Lambda$, the quark and lepton compositeness would manifest itself as a four-fermion contact interaction. The contact interaction between two quarks, a neutrino and a charged lepton is described by the Helicity-Non-Conserving-Model (HNC) [16]. The corresponding cross section is proportional to the square of the center-of-mass energy and to $\Lambda^{-4}$. This process would yield an unstructured excess, decreasing as a function of $M_{T}$ (see Equation 1), as shown in Figure 1-right. In the HNC model there is no interference of the final state with the SM W because of their different chiral structure.

Candidate events with at least one high-transverse-momentum $\left(\mathrm{p}_{\mathrm{T}}\right)$ lepton are selected using single-muon (with $\mathrm{p}_{\mathrm{T}}>40 \mathrm{GeV}$ ) and single-electron (with $\mathrm{p}_{\mathrm{T}}>80 \mathrm{GeV}$ ) triggers. The offline $\mathrm{p}_{\mathrm{T}}$ threshold of the muon (electron) channel is set to $45 \mathrm{GeV}(100 \mathrm{GeV})$ in order to be in the plateau region. Muons are reconstructed by combining information from the tracker and the muon system. The reconstruction and selection criteria include constraints on track isolation, the transverse impact parameter and the measured relative uncertainty of the muon $\mathrm{p}_{\mathrm{T}}$. Electrons are reconstructed as isolated objects in the electromagnetic calorimeter. Additional requirements are made on the 

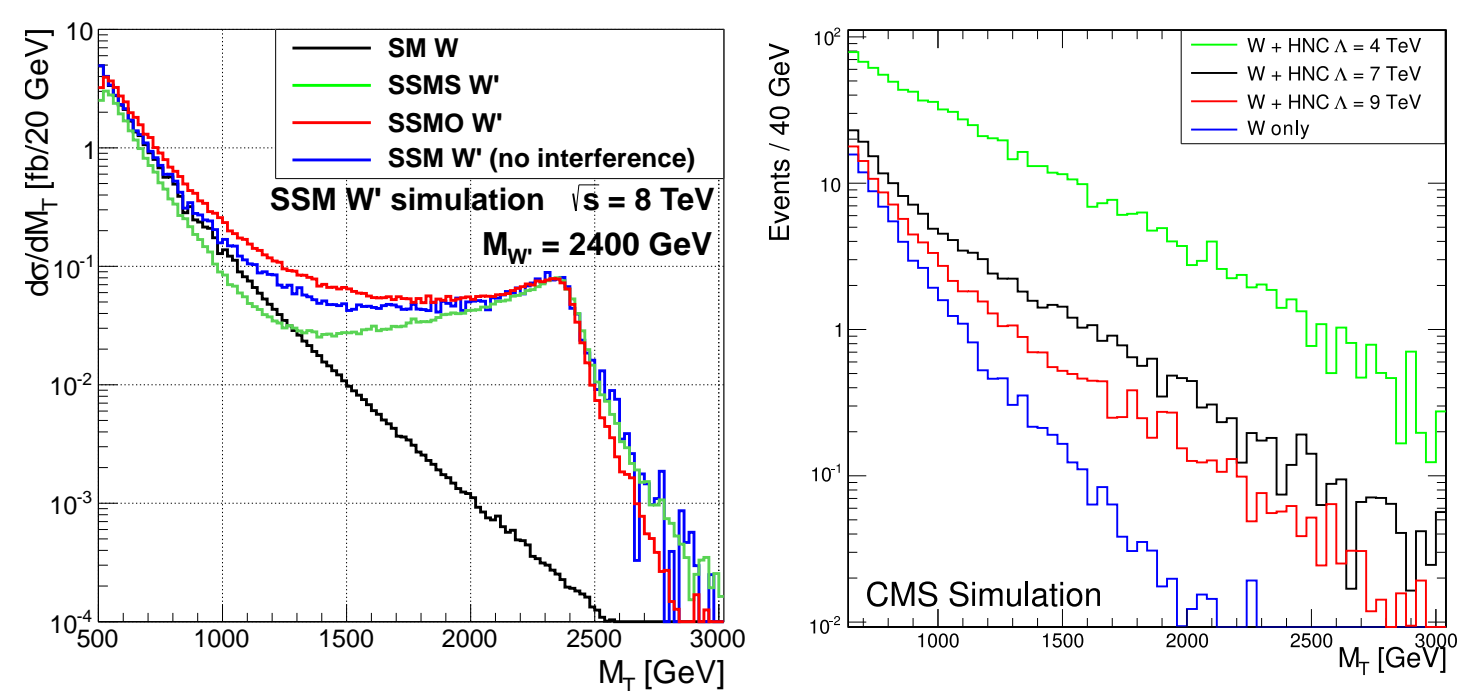

Figure 1: Left: Comparison of the different interference scenarios $(\mathrm{SSM}=$ no interference, $\mathrm{SSMO}=$ constructive interference, SSMS = destructive interference) in the $\mathrm{W}^{\prime}$ interpretation. The impact of the interference on the combined $\mathrm{W}-\mathrm{W}^{\prime}$ transverse mass spectrum is shown for the example of a $2.4 \mathrm{TeV} \mathrm{W}^{\prime}$. Additionally, in black the SM W transverse mass without the contribution of a $\mathrm{W}^{\prime}$ is shown. Right: Contact interaction (HNC model) with a lepton and missing transverse energy in the final state. Displayed is the transverse mass distribution at generator level for the main SM background $(\mathrm{W} \rightarrow \ell v)$ and three examples of CI signals with $\Lambda=4,7$, and $9 \mathrm{TeV}$.

ratio of energy deposited in the hadronic and electromagnetic calorimeter, the shower shape, and the electron track. In both channels, events with a second isolated lepton are rejected. The neutrino is not detected directly, but gives rise to experimentally observed missing transverse energy $\left(\mathrm{E}_{\mathrm{T}}^{\mathrm{miss}}\right)$. This quantity is determined using a particle-flow technique [17], an algorithm designed to reconstruct a complete list of distinct particles using all the subcomponents of the CMS detector. In $\mathrm{W}^{\prime}$ decays, as well as in the HNC model, the lepton and $\mathrm{E}_{\mathrm{T}}^{\text {miss }}$ are expected to be almost back-to-back in the transverse plane, and balanced in transverse energy. Additional kinematic criteria therefore select events with a ratio of the lepton $\mathrm{p}_{\mathrm{T}}^{\ell}$ and the $\mathrm{E}_{\mathrm{T}}^{\text {miss }}, 0.4<\mathrm{p}_{\mathrm{T}}^{\ell} / \mathrm{E}_{\mathrm{T}}^{\text {miss }}<1.5$, along with the requirement of the angular difference, $\Delta \phi_{\ell, v}>0.8 \pi$. Here, $\Delta \phi_{\ell, v}$ is the azimuthal opening angle between the charged lepton's transverse momentum $\left(\mathrm{p}_{\mathrm{T}}^{\ell}\right)$ and $\mathrm{E}_{\mathrm{T}}^{\mathrm{miss}}$ direction. The main observable in this search is the transverse mass $\mathrm{M}_{\mathrm{T}}$ of the lepton- $\mathrm{E}_{\mathrm{T}}^{\text {miss }}$ system, calculated as

$$
\mathrm{M}_{\mathrm{T}}=\sqrt{2 \cdot \mathrm{p}_{\mathrm{T}}^{\ell} \cdot \mathrm{E}_{\mathrm{T}}^{\mathrm{miss}} \cdot\left(1-\cos \Delta \phi_{\ell, v}\right)} .
$$

Several large samples of simulated events were used to evaluate signal and background efficiencies. The generated events were processed through a full simulation of the CMS detector based on GEANT4 [18, 19], a trigger emulation, and the event reconstruction chain. The selection and reconstruction efficiency is measured in data and simulation using tag-and-probe methods. Differences are accounted for by applying a scale factor to the simulation. Details about signal efficiencies, generators and cross sections can be found in [2]. The primary source of SM background 

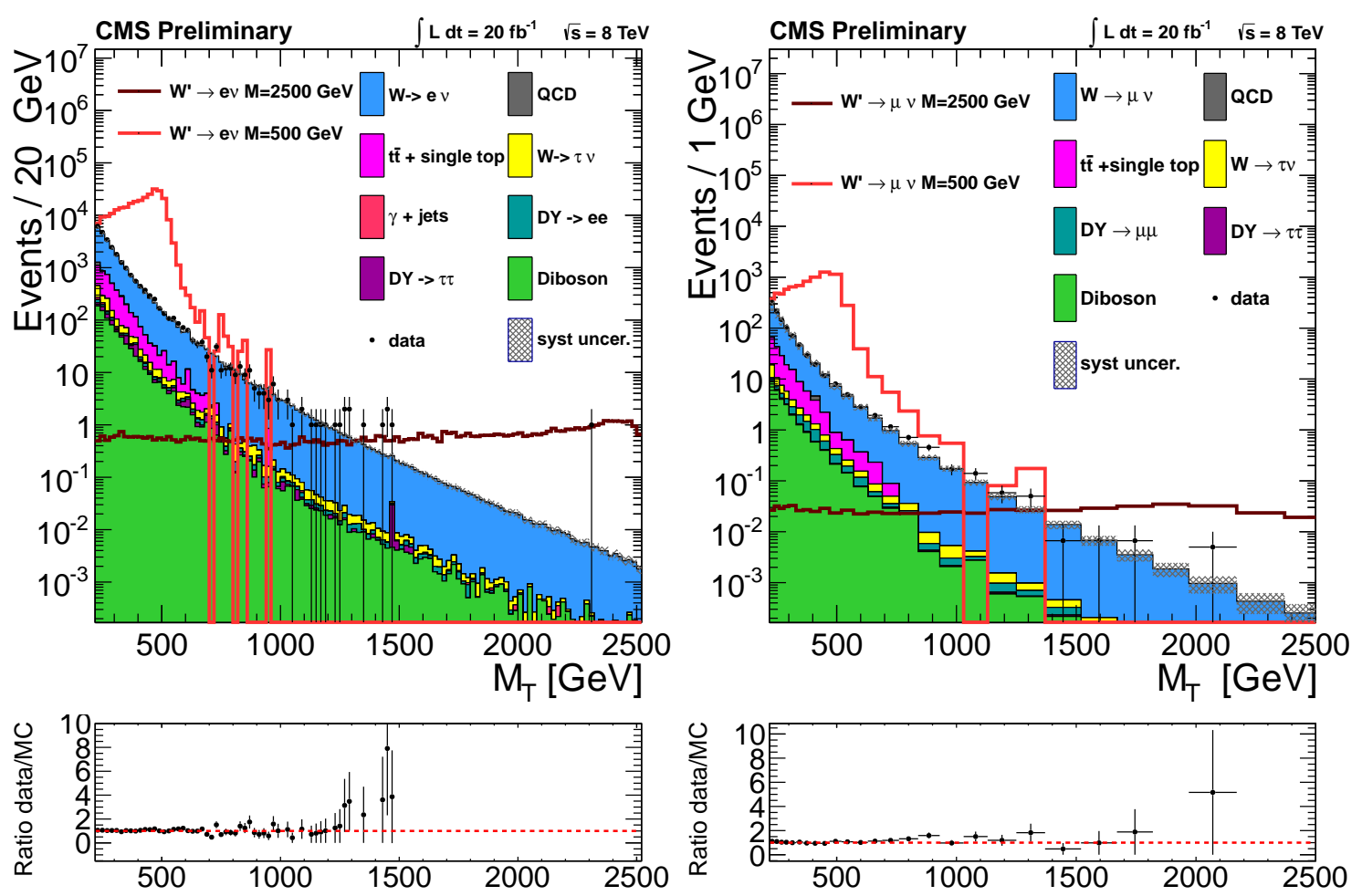

Figure 2: Observed transverse mass distributions for the electron (left) and muon (right) channels. The latter uses variable binning considering the $\mathrm{p}_{\mathrm{T}}$ dependent momentum resolution. The shaded area represents the total systematic uncertainty on the SM background. Simulated signal distributions for $\mathrm{W}^{\prime}$ masses of 0.5 and $2.5 \mathrm{TeV}$ are also shown. Detector resolution effects are included. The simulated background labeled as 'di-boson' includes WW, ZZ and WZ contributions.

is the off-peak, high transverse mass tail of $\mathrm{W} \rightarrow \ell v$ decays. Other important backgrounds arise from QCD multijet, $\mathrm{t} \overline{\mathrm{t}}$, and Drell-Yan events. Di-bosons (WW, WZ, ZZ) decaying to electrons, muons, or taus were also considered. The background is determined from simulation based on samples with high statistics at high $\mathbf{M}_{\mathrm{T}}$. The simulation of pile-up is included in all event samples by superimposing minimum bias interactions onto the simulated processes.

Uncertainties due to lepton energy or momentum resolution and scale are applied to the transverse mass spectrum, which is distorted (scaled and smeared). For the missing transverse energy, each energy deposition is assigned to its corresponding object (jet, tau, photon, electron, muon, and unclustered energy). It is then varied according to its type and from this the uncertainty on the missing transverse energy is calculated. Additional uncertainties on the integrated luminosity and the pileup determination are incorporated. The total uncertainty of the expected background is shown as grey, hatched bands in Figure 2. The largest contribution in the electron channel derives from the electron energy scale, while the muon-transverse-momentum scale constitutes the major contribution in the muon channel.

The transverse mass distributions for accepted events in the electron and muon channels are shown in Figure 2, along with two example $\mathrm{W}^{\prime}$ signals. Data are represented by black dots based on $20 \mathrm{fb}^{-1}$ of pp collision data at $\sqrt{s}=8 \mathrm{TeV}$. The various contributions of SM processes to the total 
background are shown in different colors. No significant excess has been observed in the data. The observed highest transverse mass events have $\mathrm{M}_{\mathrm{T}}=2.3 \mathrm{TeV}$ in the electron channel, and $\mathrm{M}_{\mathrm{T}}$ $=2.1 \mathrm{TeV}$ in the muon channel.

Upper limits on the production cross section times the branching fraction $\sigma_{\mathrm{W}^{\prime}} \times \mathscr{B}\left(\mathrm{W}^{\prime} \rightarrow \ell v\right)$, with $\ell=\mathrm{e}$ or $\mu$ are set. Cross section limits are derived using a Bayesian method with a uniform prior probability distribution for the signal cross section. Systematic uncertainties on the signal and background yield were included via nuisance parameters with a log-normal prior distribution. The limits are calculated using a binned likelihood. Multiple bins, as displayed in Figure 2 with $\mathrm{M}_{\mathrm{T}}>250 \mathrm{GeV}$, are considered. When combining the electron and muon channel, the uncertainties deriving from the different lepton measurements are assumed to be independent. Uncertainties due to the missing transverse energy determination, the pileup and the luminosity measurement are considered to be fully correlated.

Using the central value of the theoretical cross section times the branching fraction, the existence of a SSM W' with SM-like coupling is excluded at $95 \%$ confidence level for $\mathrm{W}^{\prime}$ masses less than $3.35 \mathrm{TeV}$ (compared with an expected limit of 3.40 TeV). Figure 3-left displays the excluded $\mathrm{W}^{\prime}$ cross section times branching ratio as a function of the $\mathrm{W}^{\prime}$ mass. To determine the limits on the $\mathrm{W}^{\prime}$ models including interference effects with the $\mathrm{W}$, the difference $\Delta \sigma$ in the $\mathrm{M}_{\mathrm{T}}$ spectrum between the $\mathrm{W}-\mathrm{W}^{\prime}$ signal sample and the $\mathrm{W}$-only sample is considered. Limits on the cross section modifier $\Delta \sigma / \Delta \sigma_{\mathrm{W}^{\prime}}$ are summarized in Table 1 .
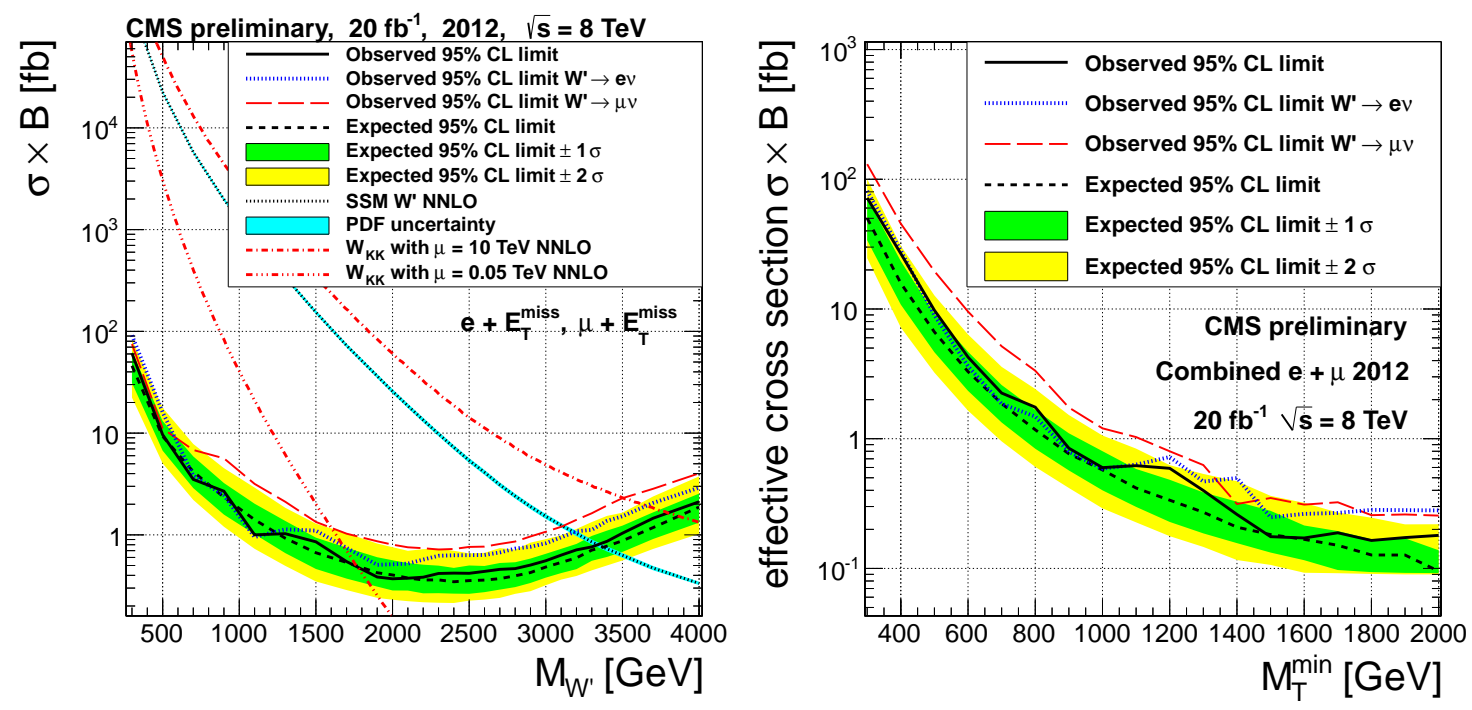

Figure 3: Left: Limits for heavy $\mathrm{W}^{\prime}$ bosons based on the 2012 data for the electron and the muon channels as well as their combination. The $\mathrm{W}^{\prime}$ mass limits are derived with a Bayesian method. The lines labeled as $\mathrm{W}_{\mathrm{KK}}$ correspond to an additional interpretation presented in [2]. Right: Upper cross section limits at 95\% confidence level on effective cross section $\sigma\left(\mathrm{W}^{\prime}\right) \times B\left(\mathrm{~W}^{\prime} \rightarrow \ell v\right)$ above a threshold $\mathrm{M}_{\mathrm{T}}^{\min }$. The one (two) sigma uncertainty bands are shown in green (yellow). Detector efficiencies are accounted for.

In the HNC contact interaction interpretation, a limit can be set on the binding energy scale $\Lambda$. The statistical interpretation is identical to the $\mathrm{W}^{\prime}$ case. The limit on $\Lambda$ is calculated to be $13.0 \mathrm{TeV}$ in the electron and $10.9 \mathrm{TeV}$ in the muon channel. No combination is attempted here since the 
compositeness substructure may differ between the channels.

Apart from the model dependent multi bin limits, a cross section limit for an unspecific signal is determined by using a single bin ranging from a lower threshold $\mathrm{M}_{\mathrm{T}}^{\min }$ to infinity, as shown in Figure 3-right.

Table 1: Summary of all exclusion limits in the electron and muon channels as well as their combinations. The interpretation labelled as SSM assumes no interference of $\mathrm{W}^{\prime}$ and SM W. In the SSMO (SSMS) model a constructive (destructive) interference is assumed. Limits on $\Lambda$ in the HNC contact interaction are given in the last rows.

\begin{tabular}{|c|c|c|c|}
\hline Model & Channel & Observed limit & Expected limit \\
\hline SSM & $\mathrm{e}$ & $\mathrm{m}_{\mathrm{W}^{\prime}}<3.20 \mathrm{TeV}$ & $\mathrm{m}_{\mathrm{W}^{\prime}}<3.25 \mathrm{TeV}$ \\
SSM & $\mu$ & $\mathrm{m}_{\mathrm{W}^{\prime}}<3.15 \mathrm{TeV}$ & $\mathrm{m}_{\mathrm{W}^{\prime}}<3.10 \mathrm{TeV}$ \\
SSM & combined & $\mathrm{m}_{\mathrm{W}^{\prime}}<3.35 \mathrm{TeV}$ & $\mathrm{m}_{\mathrm{W}^{\prime}}<3.40 \mathrm{TeV}$ \\
\hline SSMO & $\mathrm{e}$ & $\mathrm{m}_{\mathrm{W}^{\prime}}<3.60 \mathrm{TeV}$ & $\mathrm{m}_{\mathrm{W}^{\prime}}<3.60 \mathrm{TeV}$ \\
SSMO & $\mu$ & $\mathrm{m}_{\mathrm{W}^{\prime}}<3.05 \mathrm{TeV}$ & $\mathrm{m}_{\mathrm{W}^{\prime}}<3.30 \mathrm{TeV}$ \\
SSMO & combined & $\mathrm{m}_{\mathrm{W}^{\prime}}<3.80 \mathrm{TeV}$ & $\mathrm{m}_{\mathrm{W}^{\prime}}<3.80 \mathrm{TeV}$ \\
SSMS & $\mathrm{e}$ & $\mathrm{m}_{\mathrm{W}^{\prime}}<3.00 \mathrm{TeV}$ & $\mathrm{m}_{\mathrm{W}^{\prime}}<3.10 \mathrm{TeV}$ \\
SSMS & $\mu$ & $\mathrm{m}_{\mathrm{W}^{\prime}}<2.80 \mathrm{TeV}$ & $\mathrm{m}_{\mathrm{W}^{\prime}}<2.90 \mathrm{TeV}$ \\
SSMS & combined & $\mathrm{m}_{\mathrm{W}^{\prime}}<3.10 \mathrm{TeV}$ & $\mathrm{m}_{\mathrm{W}^{\prime}}<3.20 \mathrm{TeV}$ \\
\hline HNC CI & $\mathrm{e}$ & $\Lambda<13.0 \mathrm{TeV}$ & $\Lambda<13.3 \mathrm{TeV}$ \\
HNC CI & $\mu$ & $\Lambda<10.9 \mathrm{TeV}$ & $\Lambda<12.2 \mathrm{TeV}$ \\
\hline
\end{tabular}

\section{References}

[1] CMS Collaboration, The CMS experiment at the CERN LHC, Journal of Instrumentation 3 (2008), no. 08 S08004.

[2] CMS Collaboration, Search for new physics in the final states with a lepton and missing transverse energy at $\sqrt{s}=8 \mathrm{TeV}$, CMS Physics Analysis Summary CMS-PAS-EXO-12-060, (2013). http://cds.cern.ch/record/1522476.

[3] G. Altarelli, B. Mele, and M. Ruiz-Altaba, Searching for New Heavy Vector Bosons in p̄̄ Colliders, Z.Phys. C45 (1989) 109.

[4] CDF Collaboration, Search for a New Heavy Gauge Boson $W^{\prime}$ with Electron + missing $E_{T}$ Event Signature in p p collisions at $\sqrt{s}=1.96$ TeV, Phys.Rev. D83 (2011) 031102, [arXiv: 012.5145$].$

[5] D0 Collaboration, Search for $W^{\prime}$ bosons decaying to an electron and a neutrino with the Do detector, Phys.Rev.Lett. 100 (2008) 031804, [arXiv: 0710.2966 ].

[6] CMS Collaboration, Search for a heavy gauge boson $W^{\prime}$ in the final state with an electron and large missing transverse energy in pp collisions at $\sqrt{s}=7$ TeV, Phys.Lett. $\mathbf{B 6 9 8}$ (2011) 21-39, [arXiv:1012.5945].

[7] CMS Collaboration, Search for a $W^{\prime}$ boson decaying to a muon and a neutrino in pp collisions at $\sqrt{s}=7$ TeV, Phys.Lett. B701 (2011) 160-179, [arXiv:1103.0030].

[8] CMS Collaboration, Search for leptonic decays of $W^{\prime}$ bosons in pp collisions at $\sqrt{s}=7$ TeV, JHEP 1208 (2012) 023, [arXiv:1204.4764]. 
[9] CMS Collaboration, Search for new physics in final states with a lepton and missing transverse energy in pp collisions at the LHC, Phys.Rev. D87 (2013) 072005, [arXiv: 1302 . 2812].

[10] ATLAS Collaboration, Search for high-mass states with one lepton plus missing transverse momentum in proton-proton collisions at $\sqrt{s}=7 \mathrm{TeV}$ with the ATLAS detector, Phys.Lett. $\mathbf{B 7 0 1}$ (2011) 50-69, [arXiv:1103.1391].

[11] ATLAS Collaboration, Search for a heavy gauge boson decaying to a charged lepton and a neutrino in $1 \mathrm{fb}-1$ of pp collisions at $\sqrt{s}=7 \mathrm{TeV}$ using the ATLAS detector, Phys.Lett. B705 (2011) 28-46, [arXiv:1108.1316].

[12] E. Accomando, D. Becciolini, S. De Curtis, D. Dominici, L. Fedeli, et al., Interference effects in heavy W'-boson searches at the LHC, Phys.Rev. D85 (2012) 115017, [arXiv:1110.0713].

[13] T. G. Rizzo, The Determination of the Helicity of $W^{\prime}$ Boson Couplings at the LHC, JHEP 0705 (2007) 037, [arXiv:0704.0235].

[14] E. Boos, V. Bunichev, L. Dudko, and M. Perfilov, Interference between $W^{\prime}$ and $W$ in single-top quark production processes, Phys.Lett. B655 (2007) 245-250, [hep-ph / 0610080 ].

[15] H. Terazawa, M. Yasue, K. Akama, and M. Hayashi, Observable Effects of the Possible Substructure of Leptons and Quarks, Phys.Lett. B112 (1982) 387.

[16] K. Lane, F. Paige, T. Skwarnicki, and W. Womersley, Simulations of Supercollider Physics, Phys.Rept. 278 (1997) 291-371, [hep-ph/9412280].

[17] CMS Collaboration, Particle-Flow Event Reconstruction in CMS and Performance for Jets, Taus, and MET, CMS Physics Analysis Summary CMS-PAS-PFT-09-001, (2009). http://cds.cern.ch/record/1194487.

[18] GEANT4 Collaboration, GEANT4: A Simulation toolkit, Nucl.Instrum.Meth. A506 (2003) 250-303.

[19] J. Allison, K. Amako, J. Apostolakis, H. Araujo, P. Dubois, et al., Geant4 developments and applications, IEEE Trans.Nucl.Sci. 53 (2006) 270. 